\title{
Integrated Inflammatory Stress (ITIS) Model
}

\section{Bangsgaard, Elisabeth O.; Hjorth, Poul G.; Olufsen, Mette S.; Mehlsen, Jesper; Ottesen, Johnny T.}

\section{Published in:}

Bulletin of Mathematical Biology

Link to article, DOI:

$10.1007 / \mathrm{s} 11538-017-0293-2$

Publication date:

2017

Document Version

Publisher's PDF, also known as Version of record

Link back to DTU Orbit

Citation (APA):

Bangsgaard, E. O., Hjorth, P. G., Olufsen, M. S., Mehlsen, J., \& Ottesen, J. T. (2017). Integrated Inflammatory Stress (ITIS) Model. Bulletin of Mathematical Biology, 79(7), 1487-1509. https://doi.org/10.1007/s11538-0170293-2

\section{General rights}

Copyright and moral rights for the publications made accessible in the public portal are retained by the authors and/or other copyright owners and it is a condition of accessing publications that users recognise and abide by the legal requirements associated with these rights.

- Users may download and print one copy of any publication from the public portal for the purpose of private study or research.

- You may not further distribute the material or use it for any profit-making activity or commercial gain

- You may freely distribute the URL identifying the publication in the public portal 


\title{
Integrated Inflammatory Stress (ITIS) Model
}

\author{
Elisabeth O. Bangsgaard ${ }^{1}$ • Poul G. Hjorth ${ }^{1}$. \\ Mette S. Olufsen ${ }^{2}$. Jesper Mehlsen ${ }^{3}$. \\ Johnny T. Ottesen 4
}

Received: 18 September 2016 / Accepted: 11 May 2017 / Published online: 22 June 2017

(C) Society for Mathematical Biology 2017

\begin{abstract}
During the last decade, there has been an increasing interest in the coupling between the acute inflammatory response and the Hypothalamic-Pituitary-Adrenal (HPA) axis. The inflammatory response is activated acutely by pathogen- or damagerelated molecular patterns, whereas the HPA axis maintains a long-term level of the stress hormone cortisol which is also anti-inflammatory. A new integrated model of the interaction between these two subsystems of the inflammatory system is proposed and coined the integrated inflammatory stress (ITIS) model. The coupling mechanisms describing the interactions between the subsystems in the ITIS model are formulated based on biological reasoning and its ability to describe clinical data. The ITIS model is calibrated and validated by simulating various scenarios related to endotoxin (LPS) exposure. The model is capable of reproducing human data of tumor necrosis factor alpha, adrenocorticotropic hormone (ACTH) and cortisol and suggests that repeated LPS injections lead to a deficient response. The ITIS model predicts that the most extensive response to an LPS injection in ACTH and cortisol concentrations is observed in the early hours of the day. A constant activation results in elevated levels of the variables in the model while a prolonged change of the oscillations in ACTH and
\end{abstract}

$凶$ Johnny T. Ottesen

Johnny@ruc.dk

Elisabeth O. Bangsgaard

elisabethbangsgaard@gmail.com

1 Department of Applied Mathematics and Computer Science, Technical University of Denmark, Lyngby, Denmark

2 Department of Mathematics, North Carolina State University, Raleigh, NC, USA

3 Department of Coordinating Research Centre, Frederiksberg Hospital, Frederiksberg, Denmark

4 Department of Science and Environment, Roskilde University, Roskilde, Denmark 
cortisol concentrations is the most pronounced result of different LPS doses predicted by the model.

Keywords Mathematical modeling - Nonlinear ODE model · HPA axis - Acute inflammatory system $\cdot$ Immune system $\cdot$ Response to endotoxin (LPS)

\section{Introduction}

Over the years, it has become clear that the acute inflammatory response manifests itself through the HPA axis, as illustrated in Fig. 1. Cortisol has anti-inflammatory effects, and cytokines are believed to activate the HPA axis (Beishuizen and Thijs 2003; Sternberg 2006; Tracey 2002; Webster and Sternberg 2004; Yeager et al. 2009). Models of the interaction between these two subsystems will increase the understanding of the inflammatory response and lead to refined treatments of immune system disorders such as rheumatoid arthritis, Crohn's disease, atherosclerosis, diabetes and Alzheimer's disease.

\subsection{Physiological Background}

The human immune system consists of several subsystems, e.g., the skin, the acute (innate) immune system and the adaptive immune system. When a pathogenic threat or a tissue damage is detected in the body, the acute inflammatory response is initialized. The main purpose of this response is to attract phagocytic cells to eliminate the challenge (Sternberg 2006). For instance, such a pathogenic threat could be

Fig. 1 Schematic diagram of the interaction between the HPA axis and the acute inflammatory response. The main contributors to the acute inflammatory response are the phagocytic cells and cytokines, and the HPA axis consists of hypothalamus, pituitary and the adrenal secreting hormones. The interaction between the systems is that cytokines activates the HPA axis, while the hormone cortisol has an anti-inflammatory effect

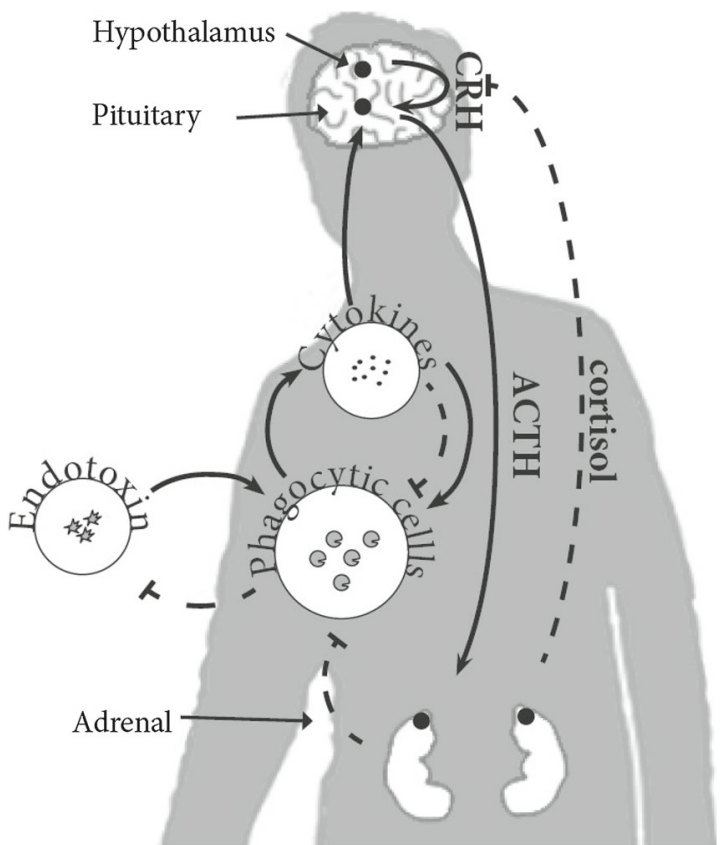


endotoxins (e.g., lipopolysaccharides, LPS) which is found in the outer membrane of gram-negative bacteria and known to activate the immune response (Amersfoort et al. 2003; Vedder et al. 1999). It is of importance that the inflammation is tightly regulated, since a too extensive response can cause further tissue damage and chronic inflammation, while an insufficient response can lead to serious infections and sepsis (Beishuizen and Thijs 2003; Sternberg 2006). Necessary components of the response regulation are cytokines, which can be classified into two principal groups: pro-inflammatory cytokines, such as interleukin-6 (IL-6) and tumor necrosis factor alpha (TNF- $\alpha$ ), and anti-inflammatory cytokines, such as interleukin-10 (IL10 ) and transforming growth factor beta (TGF- $\beta$ ) (Beishuizen and Thijs 2003; Tracey 2002). Pro-inflammatory cytokines promote inflammation, while anti-inflammatory cytokines inhibit the response to an infection.

The HPA axis is a subsystem of the immune system regulating the synthesis of the anti-inflammatory hormone cortisol (Webster and Sternberg 2004). The axis consists of the hypothalamus, the pituitary and the adrenal cortex, which releases hormones trough feedback interactions. The hypothalamus secretes corticotropin-releasing hormone (CRH) which activates the pituitary to release adrenocorticotropic hormone (ACTH). The secreted ACTH is moved by the bloodstream to the adrenal among others, where it affects the adrenal to release cortisol. Cortisol feeds back on hypothalamus and inhibits the release of $\mathrm{CRH}$ and $\mathrm{ACTH}$, leading to a down-regulation of the cortisol synthesis (Beishuizen and Thijs 2003; Walker et al. 2012; Webster and Sternberg 2004). The secretion of cortisol has been studied extensively revealing both circadian and ultradian oscillations in the concentration (Rankin et al. 2012). The release of ACTH follows a similar pattern.

The circadian rhythm of cortisol is observed in humans and shows low concentrations of cortisol in the early hours of the day, which increases during early morning hours to a maximum peak around noon, after which the overall concentrations decrease to their lower level during the night. The circadian clock is believed to be synchronized by the suprachiasmatic nuclei (SCN), located in the hypothalamus (Albrecht 2012).

Cortisol is linked to the maintenance of body homeostasis as a response to both mental and physical stress (Sternberg 2006; Webster and Sternberg 2004). Furthermore, previous studies (Jans et al. 2015a, b) have demonstrated a general, albeit transient, defect in autonomic nervous regulation of the cardiovascular system in the postoperative period of simple elective surgical procedures, and that this may be related to the inflammatory response induced by surgery. Tissue damage is expressed by damageassociated molecular patterns through pattern recognition by the Toll-like receptors protruding from the surface of macrophages (Bianchi 2007). This sensing activates transcription inducing the expression of genes initiating the inflammatory response. The inflammatory response is transmitted by pro-inflammatory cytokines in positive feed-forward loops and counteracted by anti-inflammatory cytokines, by cortisol, and by the autonomic nervous system (Tracey 2002). The interplay with the autonomic nervous system is most likely the cause of the transient dysfunction in postoperative autonomic control. 


\subsection{Modeling Background}

To our knowledge, there exists no commonly used model, which describes the interplay between the HPA axis and the acute inflammatory response, although there exist a number of models describing the systems individually.

Two illustrative examples of simple models describing the acute inflammatory response developed for studying and understanding the systemic behavior are presented by Baker et al. (2013) and Reynolds et al. (2006). The model proposed by Baker et al. (2013) is a two-dimensional model classifying all cytokines into proand anti-inflammatory, respectively. The simplicity of the model permits an analytical investigation and illustrates possible dynamics in general, although it was not compared to data. This model is analyzed by bifurcation theory in order to investigate the involvement of the pro- and anti-inflammatory cytokines in the disease rheumatoid arthritis (Baker et al. 2013). The aim of the work by Reynolds et al. (2006) was to investigate the importance of the anti-inflammatory mediators for restoring homeostasis after an infection. This model was developed as a four-dimensional model distinguishing between pathogens, phagocytic cells, a tissue damage marker and antiinflammatory mediators, representing cortisol and IL-10.

In contrast to these simple models, Chow et al. (2005) and Frank (2010) describe the response by fairly complex models in order to study and understand the detailed mechanisms of the system. The model proposed by Chow et al. (2005), which has become a standard reference, describes the acute inflammatory response to endotoxin injections in mice. The model is relative complicated and consists of 15 variables and 98 parameters. Rescaled model predictions of the concentrations of TNF- $\alpha$, IL-10, IL-6 and a NO marker are compared to experimental data for mice receiving different doses of endotoxin and induced surgery inflammation. The model fits the overall trend in data, but not quantitatively precise. The model is extended in Clermont et al. (2004) to include living bacteria. A conundrum in the paper was the need of a very slow antiinflammatory mediator which the authors suggest to be cortisol Chow et al. (2005). A model, which is perhaps in between these simple and complicated models when considering complexity, is the seven-dimensional model presented by Frank (2010). The model tends to describe the acute inflammatory response in rats receiving different doses of endotoxin. The model predictions mimic the dynamics observed in data of the cytokines IL-6, TNF- $\alpha$ and IL-10. However, there is no biological reasoning in the modeling choices.

Turning to models describing the HPA axis, the greatest difference is related to the origin of the circadian and ultradian rhythms observed in data for ACTH and cortisol. Conrad et al. (2009) induce an inclusion of a positive feedback from cortisol together with the conventional negative feedback creates the circadian rhythm, yet the model does not produce ultradian oscillations (Conrad et al. 2009). The model presented by Jelić et al. (2005) produces circadian as well as ultradian rhythms in cortisol by including an external periodic function and a positive feedback from cortisol, while the circadian rhythm of ACTH does not reflect observations (Jelić et al. 2005). Gupta et al. (2007) state that the model presented in Gupta et al. (2007) reveals bistability of the HPA axis. This model includes the glucocorticoid receptor, but it fails to produce ultradian rhythms. Another model presented by Andersen et al. (2013) also includes 
the glucocorticoid receptor revealing bistability; however, it is proved that the model is not capable of producing ultradian rhythms within a physiological range of parameter values (Andersen et al. 2013). The proposed model described by Bangsgaard and Ottesen (2016) is physiologically based and produces accurately both ultradian and circadian rhythms mainly created by nonlinearity and an external periodic function, respectively (Bangsgaard and Ottesen 2016).

Mathematical modeling of the interaction between the inflammatory response and the HPA axis is limited. To our knowledge, the first successful attempt to develop a mathematical model describing the neuroendocrine immune system was presented by Meyer-Hermann et al. (2009). This model is a six-dimensional system of ordinary differential equations. The variables represent TNF- $\alpha$, stored cholesterol, plasma cortisol and stored, local and plasma noradrenaline, respectively. The aim of the work was to describe the observed circadian rhythms in cortisol, noradrenaline and TNF$\alpha$ representing the leading operators of the endocrine, nervous and immune system, respectively. The model was fitted to data on healthy subjects as well as data on subjects suffering from rheumatoid arthritis. The model fitted the circadian rhythms in cortisol, noradrenaline and TNF- $\alpha$ well; however, the observed ultradian rhythms were omitted (Meyer-Hermann et al. 2009). Recently another important work was published by Malek et al. (2015), describing the dynamics of the HPA axis and the inflammatory cytokines IL-6 and TNF- $\alpha$ (Malek et al. 2015). Malek et al. (2015) present a model of five differential equations with two delays, containing 32 parameters and an external periodic function describing the circadian rhythm of the HPA axis. They adopt the HPA axis model from Jelić et al. (2005) discussed above. The model appears simple but is infinite-dimensional due to the delays, which in addition are relatively large (both chosen to be $10 \mathrm{~min}$ ). However, this is necessary to obtain sufficient amplitudes of the ultradian rhythms in the simulations. The aim of the work by Malek et al. (2015) is to develop a mathematical model describing the interactions between the two subsystems in order to study the bi-directional communication. The model qualitatively captures the data in Clodi et al. (2008), but the actual fit could be improved. The injection of LPS is simulated as an infusion of $2 \mathrm{IU} / \mathrm{kg}$ over $10 \mathrm{~min}$, in contrast to the study in Clodi et al. (2008).

The aim of this paper is to develop a mathematical model which can reliably predict the acute inflammatory response to endotoxin and the interplay with the hormones of the HPA axis to restore homeostasis. This paper is structured as follows. The integrated inflammatory stress (ITIS) model is presented in Sect. 2. In Sect. 3, parameter estimation of the ITIS model is carried out, and in Sect. 4, results of numerical simulations of different scenarios are presented. Finally, discussion and conclusions are provided in Sect. 5.

\section{Integrated Inflammatory Stress Model}

Inspired by the fundamental idea in the work by Malek et al. (2015), we propose a novel integrated model of the acute inflammatory response and the HPA axis. However, we are using a more accurate biological based HPA axis model (Bangsgaard and Ottesen 2016) and an adjusted model of the inflammatory response, inspired by Baker et al. 
Fig. 2 Diagram of the ITIS model. The solid arrows indicate stimulating interactions, while the dashed arrows indicate inhibiting interactions.

Endotoxin (LPS) activates the phagocytic cells which activate the cytokines. The cytokine TNF- $\alpha$ stimulates the activity of the HPA axis by activating the production and release of $\mathrm{CRH}$ and ACTH which stimulate the release of cortisol. The anti-inflammatory effects of cortisol are modulated through a stimulation of the cytokine TGF- $\beta$. The endotoxin challenge is eliminated by the phagocytic cells provided that the magnitude of the response is adequate

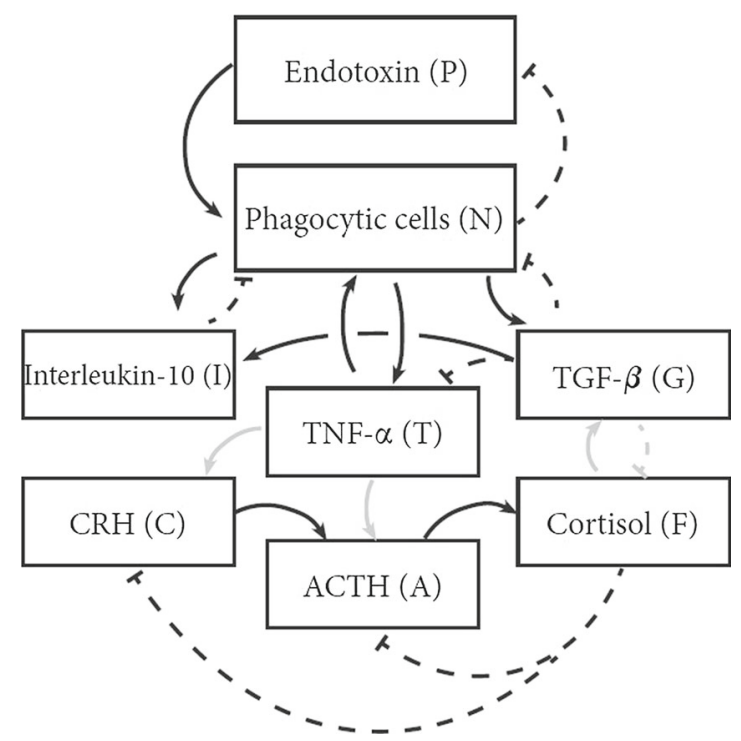

(2013), Chow et al. (2005), Frank (2010) and Reynolds et al. (2006) as point of departure. This model is developed such that each of the submodels can replicate existing data associated with the respective systems. The ITIS model contains eight timedependent variables: Endotoxin $(P)$, phagocytic cells $(N)$, pro-inflammatory cytokine: TNF- $\alpha(T)$, anti-inflammatory cytokine: IL-10 (I), Anti-inflammatory cytokine: TGF$\beta(G), C R H(C), A C T H(A)$ and cortisol $(F)$. Describing the system by nonlinear ordinary differential equations rather than infinite-dimensional delayed differential equations is a novelty compared to the model proposed by Malek et al. (2015). Another novelty is the underlying model of the HPA axis, which in this case is able to predict 24-h observations of ACTH and cortisol to a higher degree of precision than the model in Jelić et al. (2005) on which the model in Malek et al. (2015) is based. Comparing the model to the one in Meyer-Hermann et al. (2009), this model is capable of reproducing the observed ultradian oscillations in cortisol. A schematic diagram of the main interactions in the model is shown in Fig. 2. Note that removing the stimulation of $\mathrm{CRH}$ and ACTH by TNF- $\alpha$ and the interaction between cortisol and TGF- $\beta$ (indicated by the gray lines in Fig. 2) results in two decoupled models describing the acute inflammatory response and the hormone secretion of the HPA axis, respectively. For further details, see Bangsgaard (2016).

The dynamics of the HPA axis is controlled by feedback mechanisms. The secretion of CRH results in a secretion of ACTH leading to a secretion of cortisol which in turn inhibits further up-regulation of CRH and ACTH (Beishuizen and Thijs 2003; Sternberg 2006). When an endotoxin challenge is introduced to the system, the phagocytic cells are activated to eliminate the threat (Amersfoort et al. 2003; Tracey 2002). Endotoxin stimulates Toll-like receptors primarily on the surface of the macrophages or T-lymphocytes. This leads to activation of transcription factors and eventually to the production and release of cytokines. Some cytokines act as chemokines attracting T-lymphocytes responsible for inactivation of the bacteria producing the endotoxin 
while others (interleukines) activate the production of cytokines from neighboring leukocytes initiating a spreading of the inflammatory process (Dinarello 2000; Opal and DePalo 2000; Scheller et al. 2011). Macrophages which are phagocytic cells specifically release TNF- $\alpha$ as a response to endotoxin exposure (Amersfoort et al. 2003). Furthermore, activated TNF- $\alpha$ stimulates the HPA axis by up-regulating the production of CRH and ACTH (Beishuizen and Thijs 2003; Silverman et al. 2005). This results in an anti-inflammatory response modulated by TGF- $\beta$ caused by the increased secretion of cortisol (John and Buckingham 2003; Sternberg 2006; Tracey 2002). Cortisol inhibits several inflammatory processes such as cytokine production (Amersfoort et al. 2003). When the endotoxin challenge is eliminated, the system returns to a steady state.

The proposed mathematical model equations are:

$$
\begin{aligned}
\frac{\mathrm{d} P}{\mathrm{~d} t} & =-d_{1} P N \\
\frac{\mathrm{d} N}{\mathrm{~d} t} & =k_{1}\left(\left(1+k_{2} \frac{T}{h_{1}+T}\right) \frac{h_{2}}{h_{2}+G} \cdot \frac{h_{3}}{h_{3}+I}\right) P-d_{2} N \\
\frac{\mathrm{d} G}{\mathrm{~d} t} & =k_{3} N+k_{4} \frac{F}{h_{4}+F}-d_{3} G \\
\frac{\mathrm{d} T}{\mathrm{~d} t} & =\frac{N}{h_{5}+N} \cdot \frac{h_{6}^{4}}{h_{6}^{4}+G^{4}}\left(k_{5}+k_{6} \frac{T}{h_{7}+T}\right)-d_{4} T^{2} \\
\frac{\mathrm{d} I}{\mathrm{~d} t} & =b_{1}+k_{7} \frac{N^{3}}{h_{8}^{3}+N^{3}}+k_{8} \frac{G^{6}}{h_{9}^{6}+G^{6}}-d_{5} \frac{h_{10}}{h_{10}+I} I \\
\frac{\mathrm{d} C}{\mathrm{~d} t} & =b_{2}+R(t) k_{9} \frac{C}{1+k_{10} F^{2}}+k_{11} T-d_{6} C \\
\frac{\mathrm{d} A}{\mathrm{~d} t} & =k_{12} \frac{C}{1+k_{13} F}+k_{14} \frac{T^{2}}{h_{11}^{2}+T^{2}}-d_{7} A \\
\frac{\mathrm{d} F}{\mathrm{~d} t} & =k_{15} \frac{A^{2}}{1+k_{16} G}-d_{8} F,
\end{aligned}
$$

where the time-dependent external function $R(t)$ is

$$
R(t)=N_{c}\left(\frac{t_{m}^{k}}{t_{m}^{k}+\alpha^{k}} \cdot \frac{\left(T_{t}-t_{m}\right)^{l}}{\left(T_{t}-t_{m}\right)^{l}+\beta^{l}}+\epsilon\right) .
$$

The parameters $\left\{d_{i}\right\}_{i \in\{1, \ldots, 8\}}$ represent the elimination rates, $\left\{k_{j}\right\}_{j \in\{1, \ldots, 16\}}$ represent the strength of stimulation, inhibition or saturation level while $\left\{h_{l}\right\}_{l \in\{1, \ldots, 11\}}$ represent the half-saturation constants and $\left\{b_{m}\right\}_{m \in\{1,2\}}$ are basic levels. Parameter values, their units and biological interpretation appear in Appendix 6. The ITIS model is developed partly by biological reasoning and the parsimonious principle for the mathematical expressions related to data. For further details, see Bangsgaard (2016). The interpretations of the equations in the model are explained in the following. 
Equation for endotoxin $(P)$ The elimination of endotoxin is proportional to the product of the number of activated phagocytic cells and the amount of endotoxin (Asachenkov et al. 1994; Copeland et al. 2005; Harrison et al. 2011; Herald 2010; Loosbroock and Hunter 2014; Reynolds et al. 2006; Vedder et al. 1999; Zuev et al. 2006). Thus, there will be no elimination of endotoxin if there is none of the activated phagocytic cells by this modeling choice. A simple exponential decay is a common modeling choice of the elimination of endotoxin (Nieman et al. 2012). However, this choice means that elimination of endotoxin is independent of the inflammatory response.

Equation for phagocytic cells $(N)$ The number of activated phagocytic cells is strongly dependent on the presence of endotoxin. When phagocytic cells recognize bacterial components (such as endotoxin), the acute inflammatory response is initiated (Amersfoort et al. 2003; Cavaillon 1994). As long as endotoxin is present in the model, the activated phagocytic cells are further up-regulated by the pro-inflammatory TNF- $\alpha$ (Tracey 2002) and down-regulated by the anti-inflammatory mediators TGF- $\beta$ and IL-10 (Opal and DePalo 2000; Tracey 2002).

Equation for TGF- $\beta(G)$ TGF- $\beta$ is released by activated phagocytic cells (Cavaillon 1994; Opal and DePalo 2000) and modulates the anti-inflammatory effects of cortisol by a stimulation (John and Buckingham 2003).

Equation for $T N F-\alpha(T)$ The activated phagocytic cells release the pro-inflammatory cytokines TNF- $\alpha$ (Amersfoort et al. 2003; Beishuizen and Thijs 2003; Tracey 2002). TNF- $\alpha$ is auto-up-regulating (Cavaillon 1994) and inhibited by TGF- $\beta$ (Opal and DePalo 2000; Tracey 2002). The quadratic elimination rate refines the model fit of human data.

Equation for IL-10 (I) IL-10 is produced by activated phagocytic cells (Cavaillon 1994; Opal and DePalo 2000) and up-regulated by TGF- $\beta$ (Sanjabi et al. 2009). In addition, a basic level of IL-10 is present in the model, assuming that the human body is slightly anti-inflammatory when no challenges are detected. The elimination is proportional to the concentration of IL-10 for small concentrations but saturates for larger concentrations.

Equation for $\mathrm{CRH}(\mathrm{C}) \mathrm{CRH}$ released from the hypothalamus is influenced of the circadian rhythm mainly synchronized by SCN (Albrecht 2012). The auto-up-regulation of CRH is inhibited by cortisol (Beishuizen and Thijs 2003; Walker et al. 2012; Webster and Sternberg 2004). A basic level of secretion of CRH is included in the model, to ensure a positive production rate. The detection of endotoxin in the model activates the HPA axis partly through a stimulation of CRH by TNF- $\alpha$ (Beishuizen and Thijs 2003; Cavaillon 1994; John and Buckingham 2003; Silverman et al. 2005).

Equation for ACTH (A) The stimulation of ACTH by CRH is inhibited by cortisol (Beishuizen and Thijs 2003; Walker et al. 2012; Webster and Sternberg 2004). The HPA activation caused by endotoxin is modulated partly by a stimulation of ACTH by TNF- $\alpha$ (Beishuizen and Thijs 2003; John and Buckingham 2003). 
Equation for cortisol $(F)$ The secretion of cortisol is stimulated by ACTH (Beishuizen and Thijs 2003; Walker et al. 2012; Webster and Sternberg 2004). The ACTH stimulation is inhibited by TGF- $\beta$ (Liakos et al. 2003).

Equation for circadian rhythm $(R(t)) R(t)$ is an external function simulating one period of the circadian rhythm. The function models the observed circadian rhythm of the system caused by the circadian clock synchronized by the suprachiasmatic nucleus (SCN) (Albrecht 2012). The rhythm is described by a product of two Hill functions.

\section{Parameter Estimation}

Before presenting the method for parameter estimation, the data are briefly described.

\subsection{Data Description}

The data originate from a study conducted by Clodi et al. (2008), designed for investigating the impact of oxytocin on the innate immune system in humans. Data describe the response of TNF- $\alpha$, ATCH and cortisol to a endotoxin (LPS) injection of $20 \mathrm{IU} / \mathrm{kg}$ (corresponding to $2 \mathrm{ng} / \mathrm{kg}$ ) in contrast to the response affected by an additional injection of oxytocin. Only the data describing the response in the concentrations in the absence of oxytocin are considered here. Each data point is mean and standard deviation of measurements from 10 healthy men (Clodi et al. 2008).

\subsection{Parameter Selection}

The submodel describing the acute inflammatory response is fitted to data of rats receiving different doses of LPS while the submodel describing the HPA axis is fitted to data of humans in order to verify each of the submodels (Bangsgaard 2016). Using these results, the parameters introduced in the ITIS model (1)-(2) were calibrated by hand by comparing output to data. The calibrated parameters result in an adequate correspondence between the simulation of the model and data. By using parameter estimation on four selected parameters, the fit of the ITIS model is improved. The selected parameters are chosen as sensitive parameters, which vary considerably between individuals; for further discussion, see Bangsgaard (2016). The selected parameters $\delta, d_{4}$, $d_{8}$ and $k_{15}$ are among the sensitive ones without being the most sensitive. However, the quantitatively defined sensitivities alone do not describe the importance of the parameters completely. The qualitative issue of how the model output is influenced by the parameters is of paramount importance. Of course a parameter needs to be sensitive in order to have qualitative impact on the model output. A parameter may turn out to be qualitatively important although being less sensitive than most other parameters if that parameter affects the output considerably in a unique characteristic way. Thus, our choice of selected is not only based on the quantitative sensitivity criteria but also on a qualitative criteria as well. Based on this, the parameter $\delta$ describing the circadian 
phase is chosen as one of the selected parameters, since it is very important for the timing of the circadian peaks for the various curves, despite a rather small quantitative sensitivity compared to most other parameters (Bangsgaard 2016). See Appendix for the complete sensitivity analysis. The selected parameters are the elimination rate of TNF- $\alpha\left(d_{4}\right)$, the strength of the stimulation of cortisol by ACTH $\left(k_{15}\right)$, the time shifting of the phase in the circadian function $(\delta)$ and the elimination rate of cortisol $\left(d_{8}\right)$. The response of TNF- $\alpha$ varies for individuals (Yeager et al. 2009), and $d_{4}$ is a possible parameter which might change between individuals causing this difference. Investigations of the variation of $k_{15}$ and $\delta$ show that these parameters vary significantly between individuals. The significance of $d_{8}$ on the simulations has a distinct effect on the ultradian oscillations for all three hormones of the HPA axis, indicating that the system is sensitive to this parameter. The same is true for the elimination rate of CRH $\left(d_{6}\right)$; however, estimating $d_{8}$ provides a better fit to data, while keeping the concentration level of cortisol within the ranges observed from the data (Bangsgaard 2016). In contrast to cortisol, $\mathrm{CRH}$ is not easily observed in humans; thus, individual bounds for CRH are missing, suggesting that $d_{6}$ should not be selected for parameter estimation. Thus, these parameters might vary considerable between individuals compared to the other parameters, and therefore, these are chosen as selected parameters.

The selected parameters are estimated, and the result is compared to data of TNF- $\alpha$, ACTH and cortisol together with a 95\%-confidence band and a 95\%-prediction band as can be seen in Fig. 3. The confidence band gives an estimate of the uncertainty of the mean of the fitted curve, while the prediction band gives the prediction interval for new observations. The confidence and prediction band is calculated pointwise rather than simultaneously. The parameters are estimated using nlinfit, an iterative least squares method in MATLAB (R2015b).

\section{Results}

In this section, simulation results and comparison to the data presented in Sect. 3.1 are revealed. The ITIS model is simulated and analyzed for various scenarios related to endotoxin (LPS) exposure.

\subsection{Simulation of $24 \mathrm{~h}$}

Figure 4 shows a simulation of the ITIS model (1)-(2) for a 24-h interval compared to data for TNF- $\alpha(T), \operatorname{ACTH}(A)$ and cortisol $(F)$. The system is exposed to a $2 \mathrm{ng} / \mathrm{kg}$ dose of LPS $(P)$ at time $t=13.5 \mathrm{~h}$. As can be observed, the pathogenic threat is eliminated after approximately $6 \mathrm{~h}$. The system is still activated by elevated levels of phagocytic cells $(N)$, TGF- $\beta(G), \mathrm{IL}-10(I)$ and TNF- $\alpha(T)$. The elevated levels of $N$, $G, I$ and $T$ are decreasing over time (for a longer time interval than 24 h, see Fig. 5). In addition, it is seen that the oscillatory patterns for $\mathrm{CRH}, \mathrm{ACTH}$ and cortisol $(C, A$ and $F$ ) are affected. 
Fig. 3 Model prediction and data. Simulations of the ITIS model (1)-(2) presented in Sect. 2 with estimated parameters. The solid lines represent the simulation of TNF- $\alpha(T), \operatorname{ACTH}(A)$ and cortisol $(F)$, respectively, the dashed lines represent a 95\%-confidence band, and the dash-dotted line represents a 95\%-prediction band. The data are adopted from Clodi et al. (2008) (circles) and represented as a mean and standard deviation of measurements from ten subjects at each point. Time is indicated as hours after LPS injection
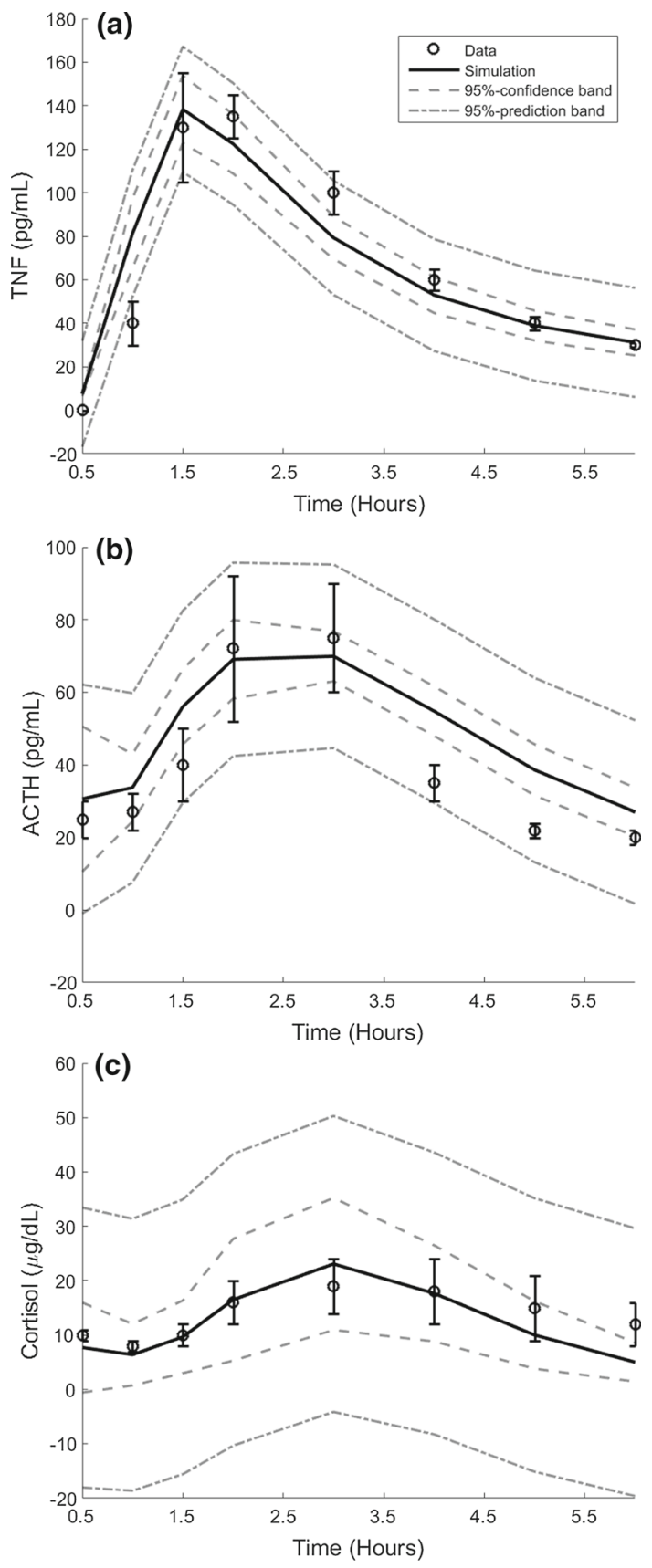

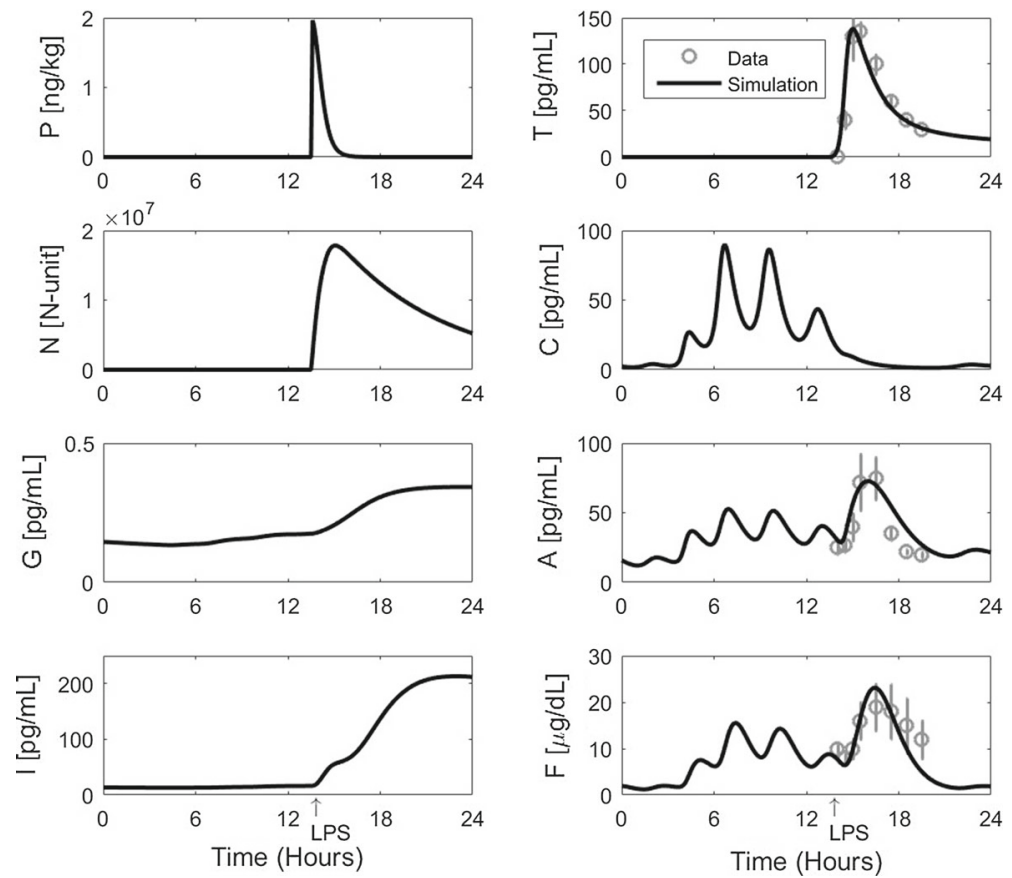

Fig. 4 Simulation of the ITIS model (1)-(2) presented in Sect. 2 over $24 \mathrm{~h}$. The solid line represents the simulation of the model, and the circles represent data. Data are adopted from Clodi et al. (2008) and are represented as a mean and standard deviation of measurements from ten subjects at each point. The LPS dose of $2 \mathrm{ng} / \mathrm{kg}$ is introduced to the system at $t=13.5 \mathrm{~h}$ as indicated at the figure

\subsection{Repeated LPS Exposure}

To study the effect on the system of repeated exposure to LPS, the ITIS model is simulated with no LPS injection, one LPS injection and two LPS injections. In Fig. 5, the three scenarios are compared for each of the eight model variables.

The injection time for the first LPS injection in the repeated simulation is the same as for the scenario with only one injection $(t=13.5)$. The second LPS injection is introduced at time $t=37.5$, i.e., $24 \mathrm{~h}$ later. The interval between the injections is chosen due to the cyclicity of the model since it is of human nature to live in a 24-h life cycle. The dose and time of the first injection is chosen according to the dose and time of injection for the calibration data. It is seen that the endotoxin $(P)$ is eliminated slower after the second LPS bolus. The injection is given before the system is returned to homeostasis, which causes a different response of the system. The response to the second injection of phagocytic cells $(N)$ is approximately less than half the magnitude of the first injection. The response of TNF- $\alpha(T)$ is also very small, compared to the first response. The response of $\mathrm{ACTH}(A)$ and cortisol $(F)$ is not detectable for the second injection.The results are similar to results found by Day et al. (2006) and illustrate the significance of the system being in homeostasis, when exposed to LPS, such that tolerance is avoided. 

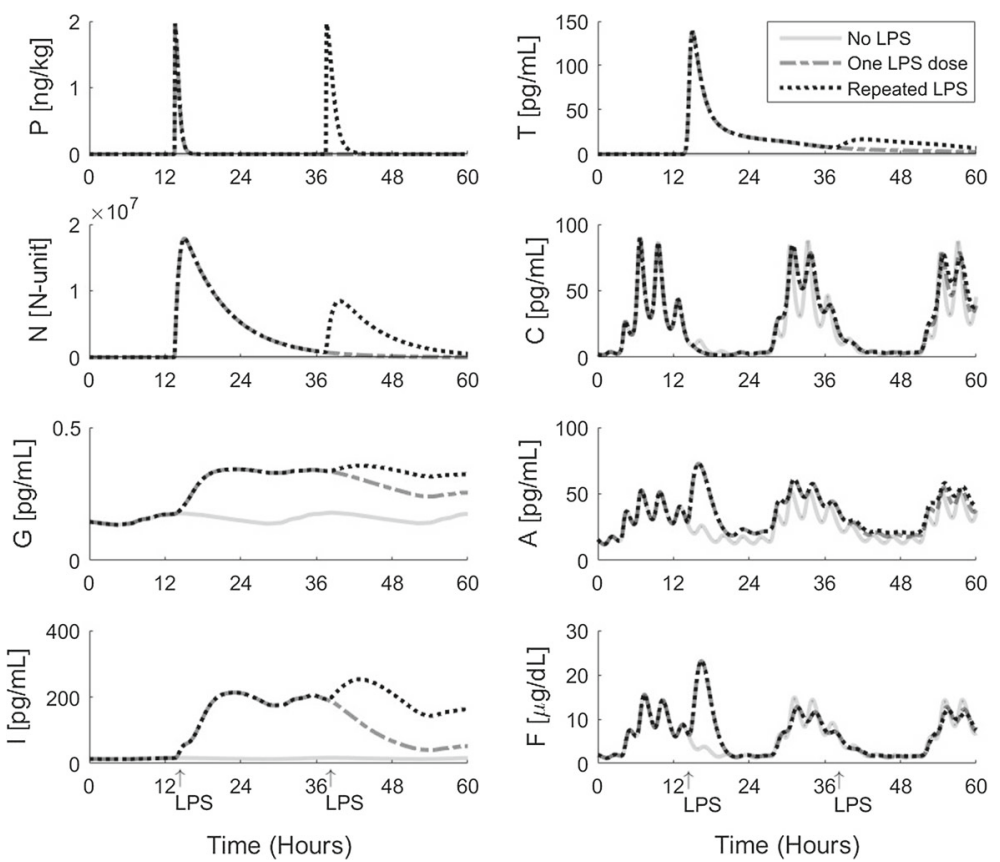

Fig. 5 Simulation of the ITIS model (1)-(2) presented in Sect. 2 over 60 h. The solid gray line represents the scenario where no LPS is introduced to the system. The dash-dotted gray line represents the scenario where LPS is introduced to the system at time $t=13.5$ while the dotted black line represents the scenario where LPS is introduced to the system at time $t=13.5$ and repeated at time $t=37.5$. The injected LPS doses are $2 \mathrm{ng} / \mathrm{kg}$ for each injection

\subsection{LPS Injections at Different Times}

To study the effect of the injection time, the ITIS model is simulated for a consistent LPS dose at different times. Figure 6 shows simulations of the ITIS model (1)-(2) for three different injection times. The LPS dose is chosen to be $2 \mathrm{ng} / \mathrm{kg}$ according to the dose for the calibration data. The time of the injections is chosen in relation to the circadian rhythm and the ultradian oscillations in CRH, ACTH and cortisol revealing that the circadian rhythm has the highest impact on the response in the model system.

In the first scenario, the LPS injection is introduced to the system at $t=6$ at the circadian peak of cortisol. The second simulation shows the response when LPS is introduced in the afternoon at $t=16$, where the circadian rhythm is declining. In addition, the response of the system is simulated for a LPS injection at the nadir of the cortisol level $(t=24.8)$. The largest responses in ACTH and cortisol are observed in the early hours of the day, while the lowest responses are observed in the afternoon, where there is a decreasing trend in the circadian rhythms of the concentrations. At the nadir of the circadian rhythm, the response of both ACTH and cortisol is remarkably high compared to the baseline at this time which is consistent with studies found in the literature (Webster and Sternberg 2004). 

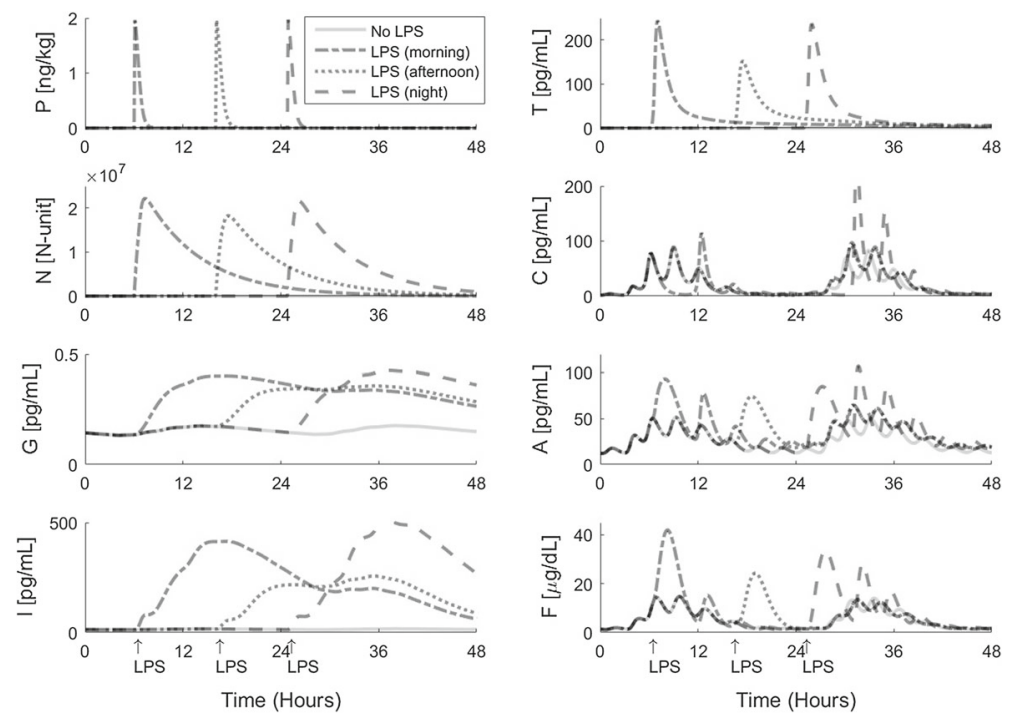

Fig. 6 Simulation of the ITIS model (1)-(2) presented in Sect. 2 over 48 h. The solid line represents the scenario where no LPS is introduced to the system. The dash-dotted line represents the scenario where LPS is introduced to the system at time $t=6$, the dotted line where LPS is introduced to the system at time $t=16$ and the dashed line where LPS is introduced at $t=24.8$. The injected LPS doses are $2 \mathrm{ng} / \mathrm{kg}$

\subsection{The Effect of Different Doses of LPS}

The system is simulated for three different doses of LPS $(0.4,2$ and $10 \mathrm{ng} / \mathrm{kg})$ at different time points, where the middle value is chosen according to the study in Clodi et al. (2008). Figure 7 shows simulations of the model for these three doses are shown for an injection time on the top of the circadian rhythm $(t=7.5)$ to illustrate some results of the study. The simulations clarify the importance within ultradian rhythms for small doses of LPS. The peak in cortisol is largest for the smallest LPS dose, when injecting on the top of the ultradian peak. The increase in cortisol for small doses of LPS has a delayed peak, compared to the response for the other doses for both injection times. The magnitude of the response in $N$ is mainly controlled by the concentration of LPS $(P)$. A large dose of LPS results in a large response of phagocytic cells, which stimulates TGF- $\beta$. The large stimulation of TGF- $\beta$ inhibits cortisol, which might be a reason, for the limited response in cortisol for large doses of LPS.

\subsection{The Effect of Baseline-Level LPS}

The effect of constant infusion of LPS on the systems response to a bolus of LPS injection is studied by introducing a baseline level of LPS of $0.1 \mathrm{ng} / \mathrm{kg}$. The response is observed for two scenarios, one where the model is exposed to an LPS injection and one where the model is not (the dose and time of injection is an LPS dose of $2 \mathrm{ng} / \mathrm{kg}$ injected at time $t=13.5$ ). This might be interpreted as a daily load from the environment, which subjects are exposed to depending on the environment, e.g., in traffic, at the work, or in the gym (Madsen 2006). This is in contrast to the previous 

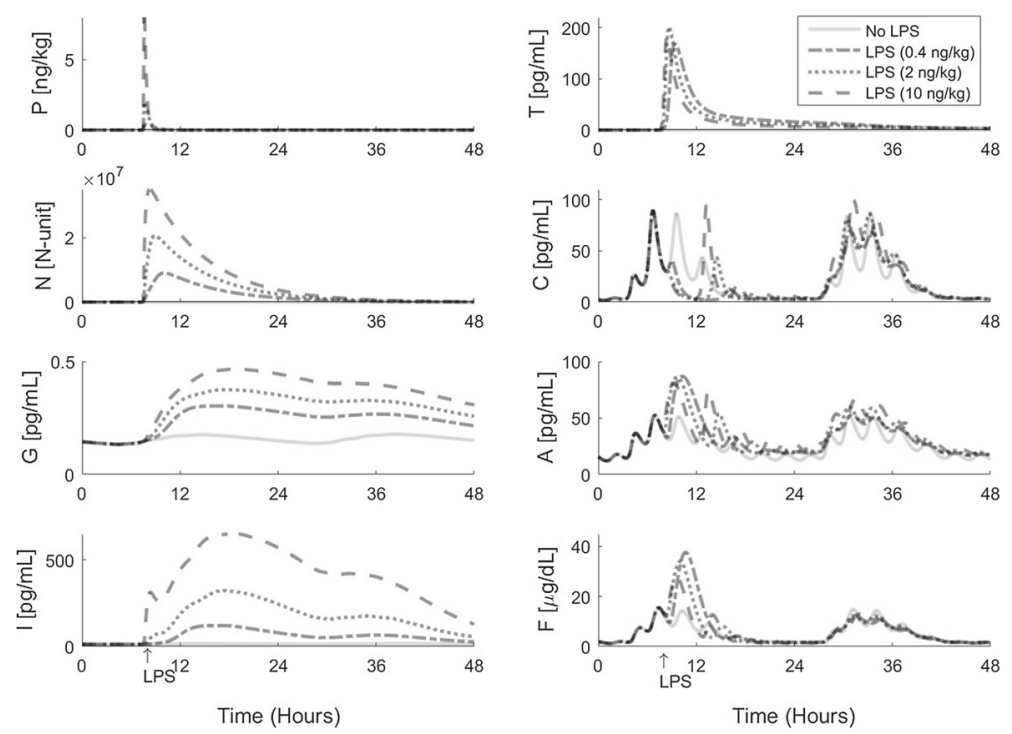

Fig. 7 Simulation of the ITIS model (1)-(2) presented in Sect. 2 over 48 h. The solid gray line represents the scenario where no LPS is introduced to the system. The dash-dotted gray line represents the scenario where a single injection of LPS (dose $0.4 \mathrm{ng} / \mathrm{kg}$ ) is introduced in the model at $t=7.5$. The dotted gray line represents the scenario where a single injection of LPS (dose $2 \mathrm{ng} / \mathrm{kg}$ ) is introduced at time $t=7.5$ and the dashed gray line represents the scenario where a LPS injection of dose $10 \mathrm{ng} / \mathrm{kg}$ is introduced at time $t=7.5$

simulations of subjects under controlled clinical circumstances (sterile conditions). An illustration of the studies is shown in Fig. 8. The constant infusion of LPS results in elevated levels of phagocytic cells, TGF- $\beta$, TNF- $\alpha$ and IL-10 compared to the simulation of the concentrations for no LPS infusion. In addition, the baseline level of LPS lowers the amplitude of the ultradian oscillations in CRH, ACTH and cortisol. The response to an injection of LPS, on the top of a baseline level of LPS, results in an absent response of TNF- $\alpha$, which is also observed for ACTH and cortisol, compared with the response to the LPS injection without a baseline level of LPS.

\section{Discussion and Conclusions}

A new model of the coupling between the acute inflammatory response and the HPA axis has been proposed and denoted the Integrated Inflammatory Stress model abbreviated the ITIS model. The ITIS model is formulated by combining two models describing the acute inflammatory response and the dynamics of the HPA axis, respectively. The models of the two subsystems were first validated separately with data and then coupled to form the novel model.

The coupling mechanisms describing the interactions between the subsystems in the models have been formulated partly by biological reasoning using the parsimonious principle and partly by fitting the ITIS model to a mean of human data measured on ten individuals exposed to endotoxin. The measured data contain information for the concentrations of TNF- $\alpha$, ACTH and cortisol after exposure of LPS dose $2 \mathrm{ng} / \mathrm{kg}$, i.e., 

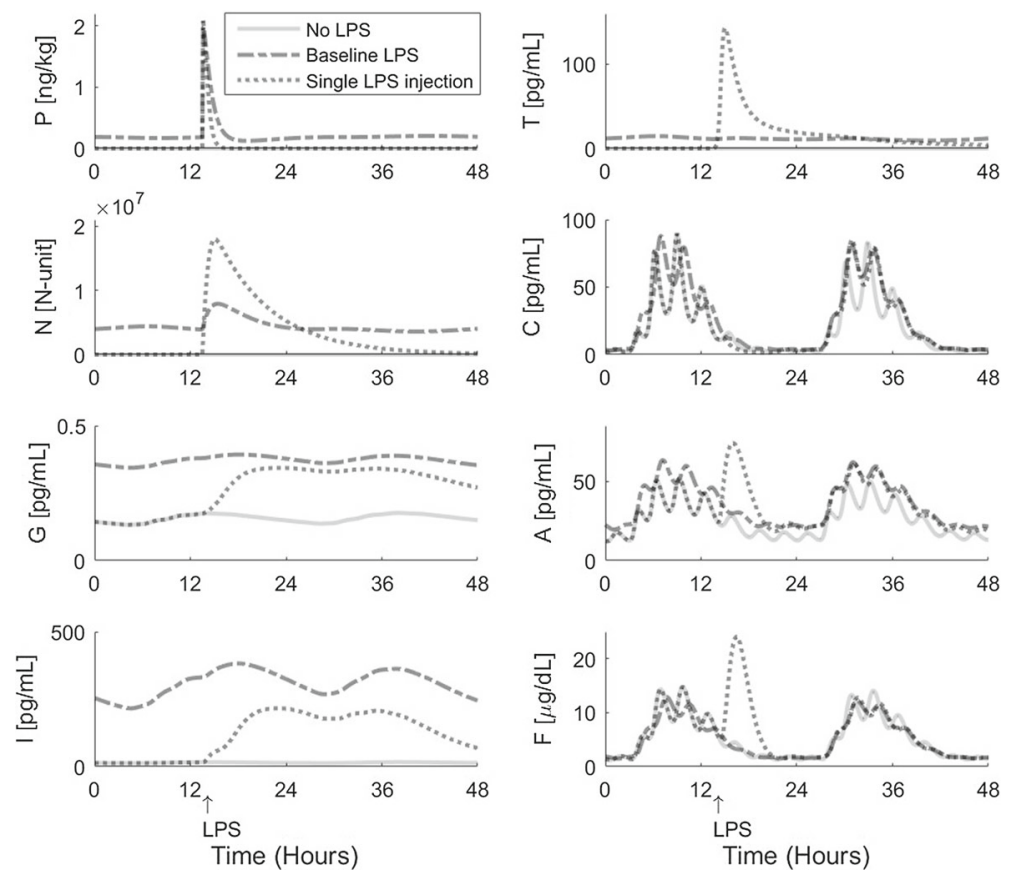

Fig. 8 Simulation of the ITIS model (1)-(2) presented in Sect. 2 over 48 h. The solid gray line represents the scenario where no LPS is introduced to the system. The dotted gray line represents the scenario where a single injection of LPS is introduced in the model at $t=13.5$, whereas the dash-dotted gray line represents the scenario where a basis level of LPS is infused in the model and a single LPS injection is introduced at time $t=13.5$ on top of the basis level. The injected LPS doses are $2 \mathrm{ng} / \mathrm{kg}$ for the single LPS injections, and the basis level of LPS is $0.1 \mathrm{ng} / \mathrm{kg}$

only three of the eight variables in the ITIS model. Four of the parameters in the ITIS model were estimated using a least squares method. The parameters $d_{4}$ (the elimination of TNF- $\alpha$ ), $k_{15}$ (the strength of the ACTH stimulation of cortisol), $\delta$ (the time shifting of the phase in the circadian function) and $d_{8}$ (the elimination rate of cortisol) are chosen as the parameters to estimate, because these vary noticeably between individuals. Comparing the residual sum of squares to the model proposed by Malek et al. (2015), the value is decreased from 13,515 to 3646 which corresponds to a $73 \%$ decrease. Additional data might help to validate the ITIS model and the simulated response to LPS. The ITIS model is described by nonlinear ordinary differential equations, which is a novelty compared to the infinite-dimensional delayed differential model presented by Malek et al. (2015).

The ITIS model has been numerically simulated for various scenarios: repeated LPS injections, different times of LPS injection, injections of different LPS doses and the effect of an LPS injection under the influence of constant LPS infusion. The simulations reveal the importance of maintaining homeostasis to obtain the most effective responses to invading pathogens and also the impact of the oscillations in cortisol. The study of the ITIS model for repeated LPS injections shows the significance of the system being in homeostasis when exposed to LPS. Thus, the ITIS model suggests that 
repeated LPS injections lead to development of tolerance which might cause a deficient immune response. Furthermore, the ITIS model suggests that the most extensive response in ACTH and cortisol concentrations are observed in the early hours of the day, which is consistent with the literature (Webster and Sternberg 2004). The most pronounced variation in the responses of the ITIS model to different doses of LPS is the prolonged changes in the oscillations of CRH, ACTH and cortisol. The concentrations of phagocytic cells, TGF- $\beta$ and IL-10 are increased significantly in accordance with the increment in LPS doses. The study of the ITIS model where the effect of a baseline level LPS is investigated suggests that a constant activation of the immune system results in elevated levels of the model variables which lead to an insufficient response to an LPS injection.

The ITIS model describes the response of the acute inflammatory system to an LPS injection. Even though LPS activates the inflammatory system, it is not able to grow and it will not be active, and therefore, it only serves as an approximation of the real world. Future development of this model could include these features by introducing possible growth in the equation for endotoxin and possibly tissue damage. However, this will increase the complexity of the model.

Eventually, the ITIS model may help in understanding the coupling between the acute inflammatory response and the HPA axis and possibly be used as a tool in the treatment of diseases involving the immune system.

\section{Appendix}

\subsection{Parameter Values}

The parameter values used for the simulations of the ITIS model (1)-(2) presented in Sect. 2 and the biological interpretation are shown in Table 1.

\subsection{Sensitivity Analysis}

The relative sensitivities of the parameters in the ITIS model are calculated to investigate the quantitative sensitivity and the robustness of the results of the model output. The relative sensitivity of a model output $y_{i}$ to the model parameters $\theta_{j}$ where $j=1, \ldots, q$ can be calculated from the sensitivity matrix

$$
S_{i}^{\text {relative }}=\left[\begin{array}{ccc}
\frac{\theta_{1}}{y_{i}} \frac{\partial y_{i}}{\partial \theta_{1}}\left(t_{i 1}\right) & \cdots & \frac{\theta_{q}}{y_{i}} \frac{\partial y_{i}}{\partial \theta_{q}}\left(t_{i 1}\right) \\
\vdots & \ddots & \vdots \\
\frac{\theta_{1}}{y_{i}} \frac{\partial y_{i}}{\partial \theta_{1}}\left(t_{i k_{i}}\right) & \cdots & \frac{\theta_{q}}{y_{i}} \frac{\partial y_{i}}{\partial \theta_{q}}\left(t_{i k_{i}}\right)
\end{array}\right]
$$

for each of the variables $i$ in the model, where $t_{i j}$ is the $k_{i}$ instance of the $j$ th measurement and $y_{i} \neq 0$. To compare the sensitivities, the two-norm of each column can be calculated and used as a time independent measure for each of the parameters. 


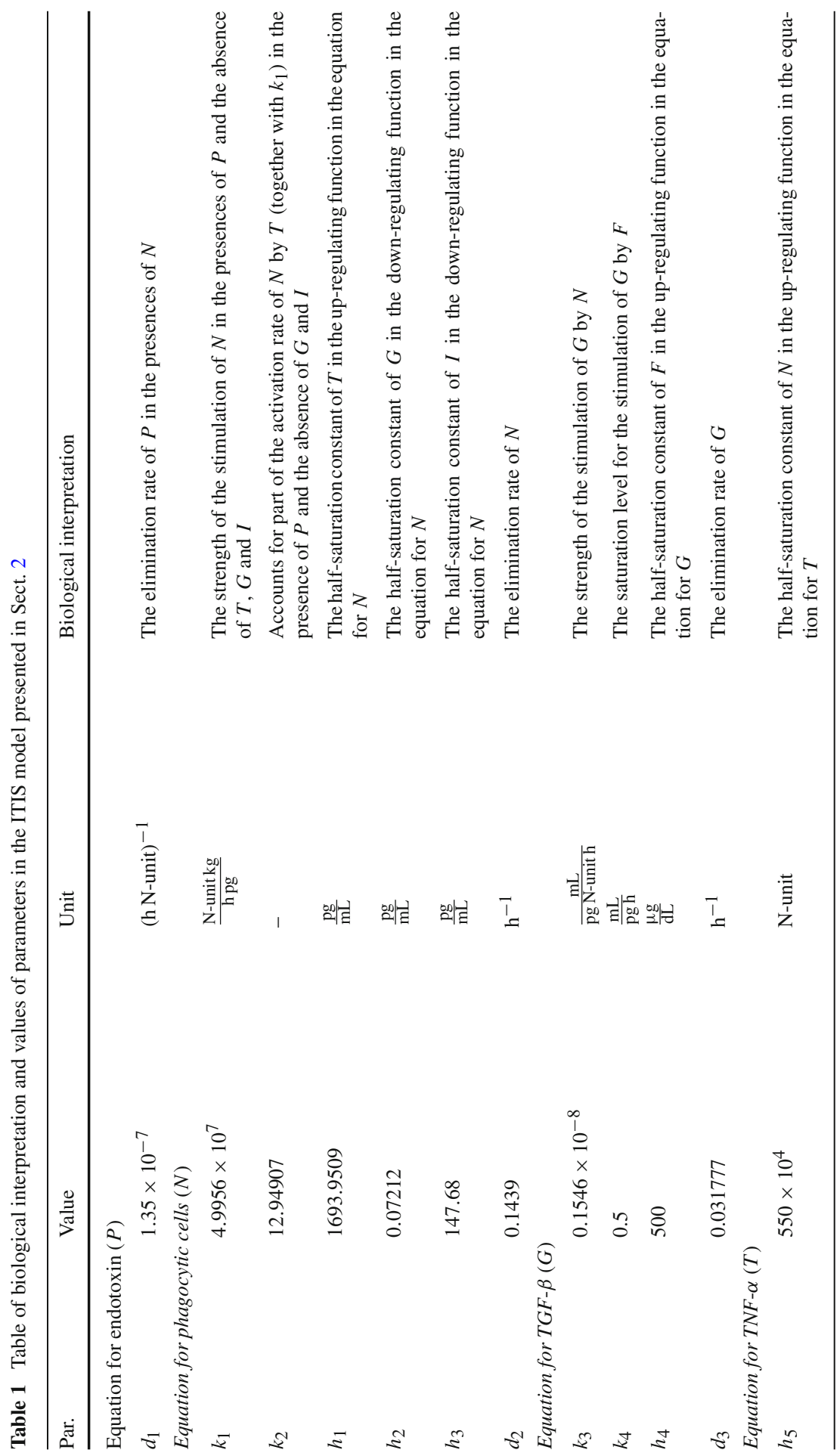




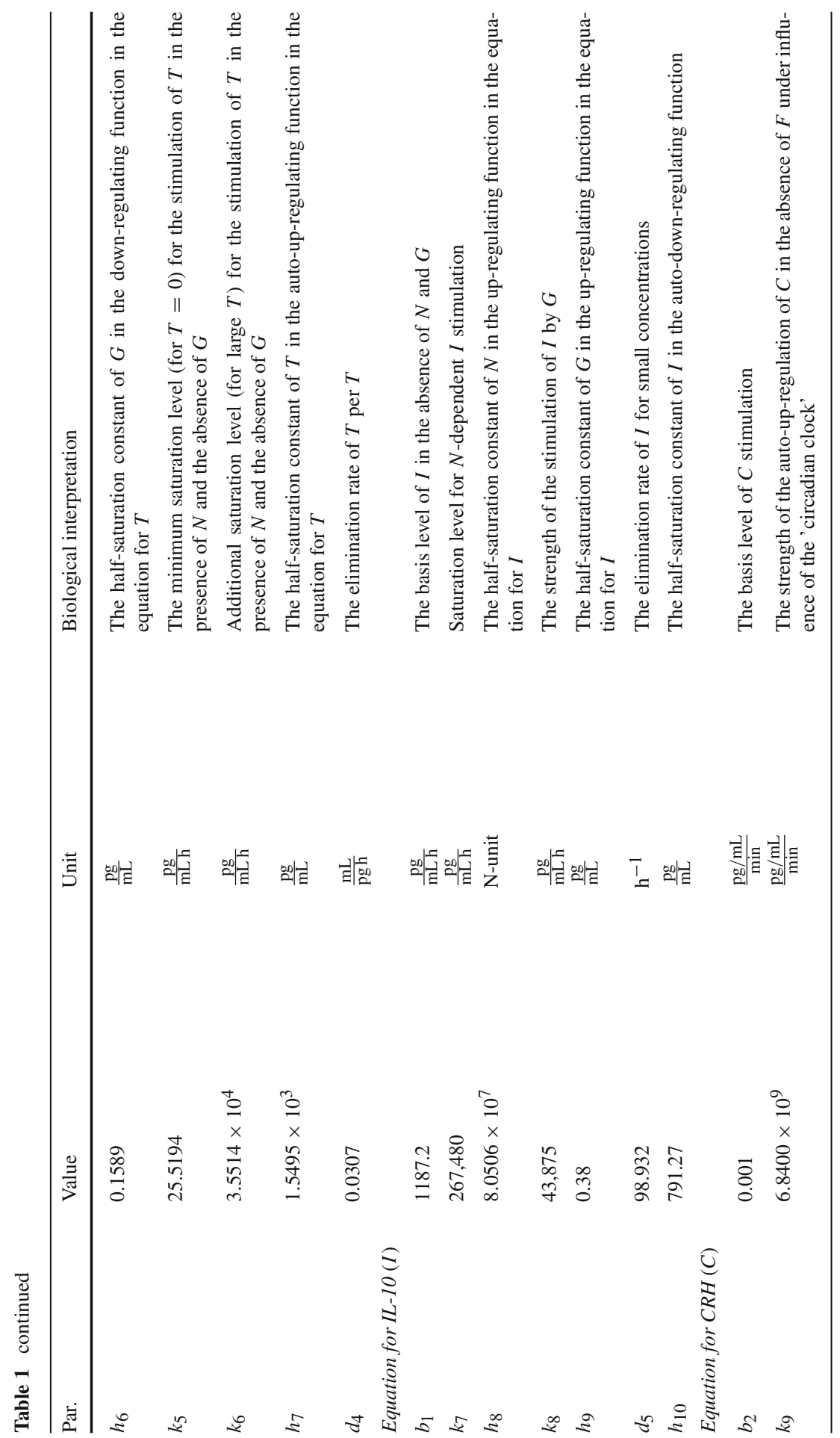



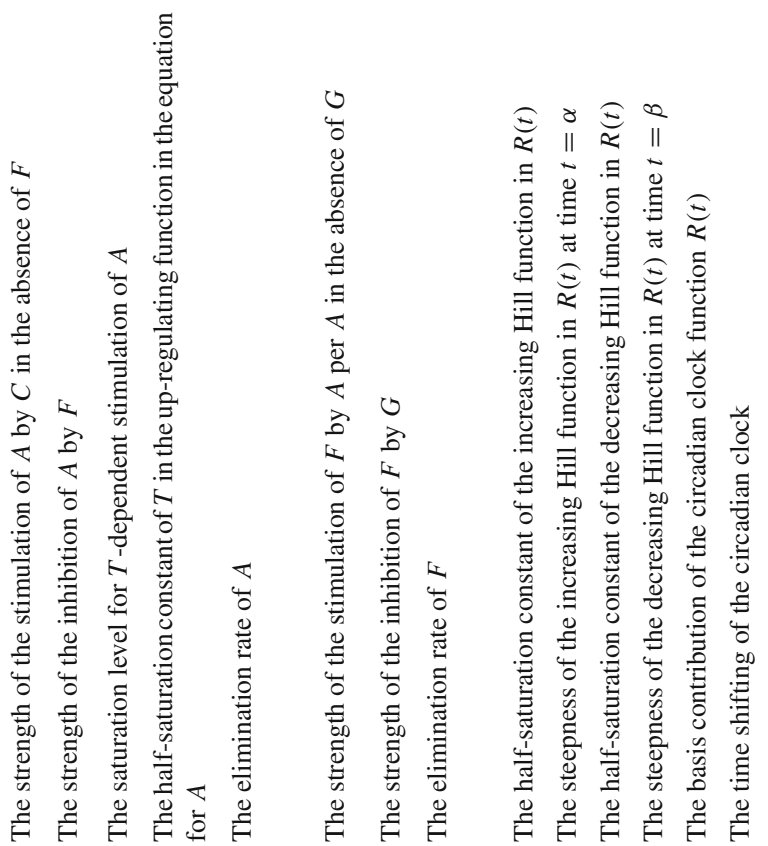

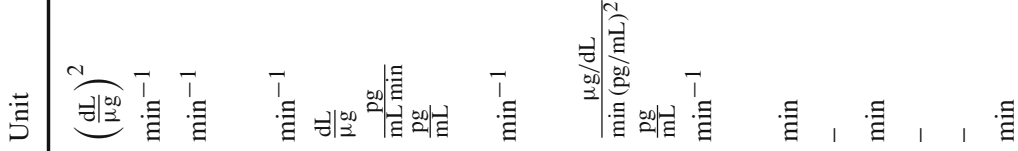

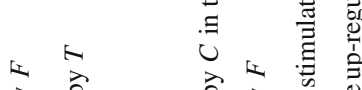

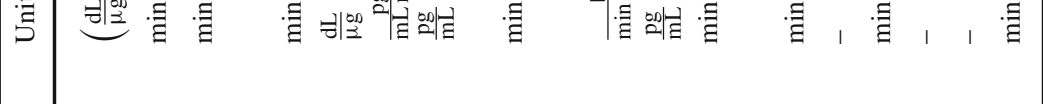

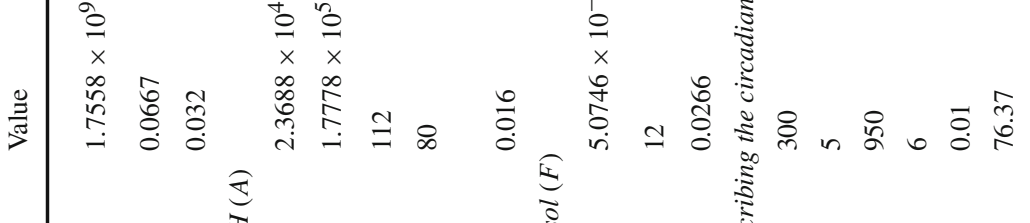

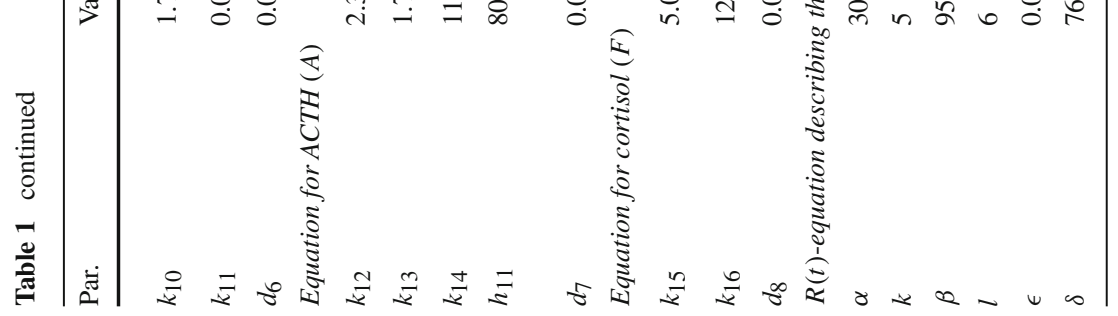




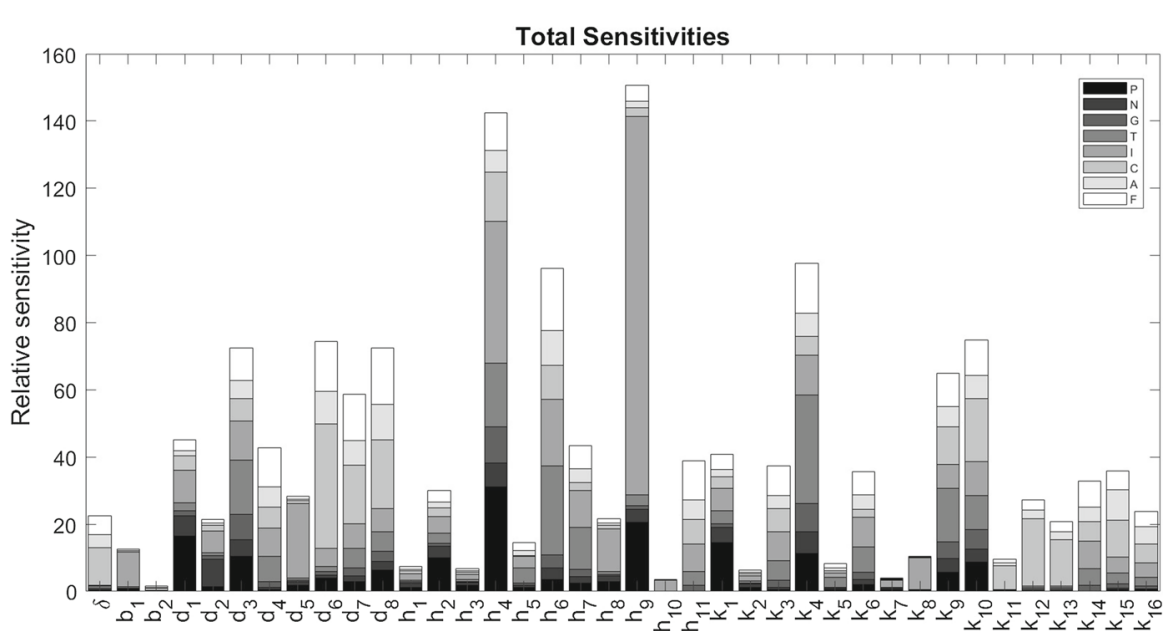

Fig. 9 Histogram of the relative sensitivities of the parameters in the ITIS model

A histogram stacking the relative sensitivities for the variables in the ITIS model is shown in Fig. 9.

\section{References}

Albrecht U (2012) Timing to perfection: the biology of central and peripheral circadian clock. Neuron 74(2):246-260. doi:10.1016/j.neuron.2012.04.006

Amersfoort ESV, Berkel TJCV, Kuiper J (2003) Receptors, mediators, and mechanisms involved in bacterial sepsis and septic shock. Clin Microbiol Rev 16(3):379-414. doi:10.1128/CMR.16.3.379-414.2003

Andersen M, Vinther F, Ottesen JT (2013) Mathematical modeling of the hypothalamic-pituitary-adrenal gland (HPA) axis, including hippocampal mechanisms. Math Biosci 246(1):122-138. doi:10.1016/j. mbs.2013.08.010

Asachenkov A, Marchuk G, Mohler R, Zuev S (1994) Disease dynamics. Birkhäuser, Basel

Baker M, Denman-Johnson S, Brook BS, Gaywood I, Owen MR (2013) Mathematical modelling of cytokine-mediated inflammation in rheumatoid arthritis. Math Med Biol 30(4):311-337. doi:10.1093/ imammb/dqs026

Bangsgaard EO (2016) Mathematical modelling of the dynamic role of the HPA axis in the immune system. Master's thesis, Technical University of Denmark, Department of Applied Mathematics and Computer Science. Supervisor: Poul G. Hjorth, pghj@dtu.dk, DTU Compute

Bangsgaard EO, Ottesen JT (2016) Patient specific modeling of the HPA axis related to clinical diagnosis of depression. Math Biosci. doi:10.1016/j.mbs.2016.10.007

Beishuizen A, Thijs LG (2003) Reviews: endotoxin and the hypothalamo-pituitary-adrenal (HPA) axis. Innate Immun 9(1):3-24. doi:10.1177/09680519030090010101

Bianchi M (2007) DAMPs, PAMPs and alarmins: all we need to know about danger. J Leukoc Biol 81:1-5. doi:10.1177/09680519030090010101

Cavaillon JM (1994) Cytokines and macrophages. Biomed Pharmacother 48:445-453

Chow CC, Clermont G, Kumar R, Lagoa C, Tawadrous Z, Gallo D, Betten B, Bartels J, Constantine G, Fink MP, Billiar TR, Vodovotz Y (2005) The acute inflammatory response in diverse shock states. Shock 24(1):74-84. doi:10.1097/01.shk.0000168526.97716.f3

Clermont G, Bartels J, Kumar R, Constantine G, Vodovotz Y, Chow C (2004) In silico design of clinical trials: a method coming of age. Crit Care Med 32(10):2061-2070

Clodi M, Vila G, Geyeregger R, Riedl M, Stulnig TM, Struck J, Luger TA, Luger A (2008) Oxytocin alleviates the neuroendocrine and cytokine response to bacterial endotoxin in healthy men. Am J Physiol Endocrinol Metab 295:686-691 
Conrad M, Hubold C, Fischer B, Peters A (2009) Modeling the hypothalamus-pituitary-adrenal system: homeostasis by interacting positive and negtive feedback. J Biol Phys 35:149-162. doi:10.1007/ s10867-009-9134-3

Copeland S, Warren HS, Lowry SF, Calcano SE, Remick D (2005) Acute inflammatory response to endotoxin in mice and humans. Clin Diagn Lab Immunol 12(1):60-67. doi:10.1128/CDLI.12.1.60-67. 2005

Day J, Rubin J, Vodovotz Y, Chow C, Reynolds A, Clermont G (2006) A reduced mathematical model of the acute inflammatory response II. Capturing scenarios of repeated endotoxin adminstration. J Theor Biol 242:237-256. doi:10.1016/j.jtbi.2006.02.015

Dinarello CA (2000) Proinflammatory cytokines. Chest 118(2):503-508

Frank DO (2010) Derivation and parameter estimation of a reduced mathematical model of acute inflammatory response to endotoxin challenge. Ph.D. thesis, North Carolina State University

Gupta S, Aslakson E, Gurbaxani BM, Vernon SD (2007) Inclusion of the glucocorticoid receptor in a hypothalamic pituitary adrenal axis model reveals bistability. Theor Biol Med Model. doi:10.1186/ 1742-4682-4-8

Harrison DG, Guzik TJ, Lob HE, Madhur MS, Marvar PJ, Thabet SR, Vinh A, Weyand CM (2011) Inflammation, immunity, and hypertension. Hypertension 75:132-140. doi:10.1161/HYPERTENSIONAHA. 110.163576

Herald MC (2010) General model of inflammation. Bull Math Biol. doi:10.1007/s11538-009-9468-9

Jans $\varnothing$, Brinth L, Kehlet H, Mehlsen J (2015a) Decreased heart rate variability responses during early postoperative mobilization-an observational study. BMC Anesthesiol. doi:10.1186/s12871-015-0099-4

Jans Ø, Mehlsen J, Kjærgaard-Andersen P, Husted H, Solgaard S, Josiassen J, Lunn TH, Kehlet H (2015b) Oral midodrine hydrochloride for prevention of orthostatic hypotension during early mobilization after hip arthroplasty: a randomized, double-blind, placebo-controlled trial. J AM Soc Anesthesiol 123(6):1292-1300. doi:10.1097/ALN.0000000000000890

Jelić S, ČupićŽ, Kolar-Anić L (2005) Mathematical modeling of the hypothalamic-pituitary-adrenal system activity. Math Biosci 197:173-187. doi:10.1016/j.mbs.2005.06.006

John CD, Buckingham JC (2003) Cytokines: regulation of the hypothalamo-pituitary-adrenocortical axis. Curr Opin Pharmacol 3(1):78-84. doi:10.1016/S1471-4892(02)00009-7

Liakos P, Lenz D, Bernhardt R, Feige JJ, Defaye G (2003) Transforming growth factor $\beta 1$ inhibits aldosterone and cortisol production in the human adrenocortical cell line NCI-H295R through inhibition of CYP11B1 and CYP11B2 expression. J Endocrinol 176:69-82

Loosbroock C, Hunter KW (2014) Inhibiting TNF-signaling does not attenuate induction of endotoxin tolerance. J Inflam Res 7:159-167. doi:10.2147/JIR.S75037

Madsen AM (2006) Airbone endotoxin in different background environments and seasons. Ann Agric Environ Med 13:81-86

Malek H, Ebadzadeh MM, Safabakhsh R, Razavi A, Zaringhalam J (2015) Dynamics of the HPA axis and inflammatory cytokines: insights from mathematical modeling. Comput Biol Med 67:1-12. doi:10. 1016/j.compbiomed.2015.09.018

Meyer-Hermann M, Figge MT, Straub RH (2009) Mathematical modeling of the circadian rhythm of key neuroendocrine-immune system players in rheumatoid arthritis. Arthritis Rheum 60:2585-2594. doi:10.1002/art.24797

Nieman G, Brown D, Sarkar J, Kubiak B, Ziraldo C, Dutta-Moscato J, Vieau C, Barclay D, Gatto L, Maier K, Constantine G, Billiar TR, Zamora R, Mi Q, Chang S, Vodovotz Y (2012) A two-compartment mathematical model of endotoxin-induced inflammatory and physiologic alterations in swine. Crit Care Med 40(4):1052-1063. doi:10.1097/CCM.0b013e31823e986a

Opal SM, DePalo VA (2000) Anti-inflammatory cytokines. Chest 117(4):1162-1172

Rankin J, Walker JJ, Windle R, Lightman SL, Terry JR (2012) Characterizing dynamic interactions between ultradian glucocorticoid rhythmicity and acute stress using the phase response curve. PLoS ONE. doi:10.1371/journal.pone.0030978

Reynolds A, Rubin J, Clermont G, Day J, Vodovotz Y, Ermentrout GB (2006) A reduced mathematical model of the acute inflammatory response: I. Derivation of model and analysis of anti-inflammation. J Theor Biol 242(1):220-236. doi:10.1016/j.jtbi.2006.02.016

Sanjabi S, Zenewicz LA, Kamanaka M, Flavell RA (2009) Anti-inflammatory and pro-inflammatory roles of TGF- $\beta$, IL-10, and IL-22 in immunity and autoimmunity. Curr Opin Pharmacol 9:447-453. doi:10. 1016/j.coph.2009.04.008 
Scheller J, Chalaris A, Schmidt-Arras D, Rose-John S (2011) The pro- and anti-inflammatory properties of the cytokine interleukin-6. BBA-Mol Cell Res 1813(5):878-888

Silverman MN, Pearce BD, Biron CA, Miller AH (2005) Immune modulation of the hypothalamicpituitary-adrenal (HPA) axis during viral infection. Viral Immunol 18(1):41-78. doi:10.1089/vim. 2005.18.41

Sternberg EM (2006) Neural regulation of innate immunity: a coordinated nonspecific host response to pathogens. Nat Rev Immunol 6:318-328. doi:10.1038/nri1810

Tracey KJ (2002) The inflammatory reflex. Nature 420:853-859. doi:10.1038/nature01321

Vedder H, Schreiber W, Yassouridis A, Gudewill S, Galanos C, Pollmcher T (1999) Dose-dependence of bacterial lipopolysaccharide (LPS) effects on peak response and time course of the immune-endocrine host response. Inflam Res 48:67-74. doi:10.1007/s000110050408

Walker JJ, Spiga F, Waite E, Zhao Z, Kershaw Y, Terry JR, Lightman SL (2012) The origin of glycocorticoid hormone oscillations. PLoS Biol 10(6):69-82. doi:10.1371/journal.pbio.1001341

Webster JI, Sternberg EM (2004) Role of the hypothalamic-pituitary-adrenal axis, glucocorticoids and clucocorticoid receptors in toxic sequelae of exposure to bacterial and viral products. J Endocrinol 181:207-221. doi:10.1677/joe.0.1810207

Yeager MP, Rassias AJ, Pioli PA, Beach ML, Wardwell K, Collins JE, Lee HK, Guyre PM (2009) Pretreatment with stress cortisol enhances the human systemic inflammatory response to bacterial endotoxin. Crit Care Med. doi:10.1097/CCM.0b013e3181a592b3

Zuev SM, Kingsmore SF, Gessler DDG (2006) Sepsis progression and outcome: a dynamical model. Theor Biol Med Model. doi:10.1186/1742-4682-3-8 